\title{
MS06-P03 | Structural Studies of Temperate Phage Genetic Switches
}

Varming, Anders (Department of Chemistry, University of Copenhagen, Copenhagen $\varnothing$, DNK); Rasmussen, Kim Krighaar (University of Copenhagen, Copenhagen $\varnothing$, DNK); Kilstrup, Mogens (Technical University of Denmark, Kgs Lyngby, DNK); Hammer, Karin (Technical University of Denmark, Kgs Lyngby, DNK); Ringkjøbing Jensen, Malene (CNRS, IBS, Grenoble, FRA); Ingmer, Hanne (University of Copenhagen, Frederiksberg, DNK); Lo Leggio, Leila (University of Copenhagen, Copenhagen $\varnothing$, DNK)

Temperate bacteriophages (TPs) may alternatively enter a lytic or a lysogenic cycle and have been shown to play a major role in the horizontal gene transfer of virulence and resistance genes between bacterial populations. The choice of lytic or lysogenic cycle in TPs is controlled by regulatory proteins and operator sites on the DNA creating a bi-stable genetic switch.

Here we present our work on the lysogeny switches of lactococcal TP901-1 phage and the staphylococcal phi13 phage, controlled by the $\mathrm{Cl}$ repressor and the MOR anti-repressor.

We have taken an integrative structural biology approach to characterize the proteins and their interactions with DNA and each other, using a wide range of techniques, including crystallography, SAS, CD and NMR spectroscopy, ITC, DSF, MS, gel filtration and EMSA. We have previously structurally characterized $\mathrm{Cl}$ repressor interaction with DNA through the N-terminal domain (NTD), the Cl C-terminal dimerization domain and the hexameric full-length $\mathrm{Cl}$ (Pedersen et al, 2008; Frandsen et al, 2013, Rasmussen et al, 2016, Rasmussen et al, 2018). Recently we have characterized the role of the flexible linker between the $\mathrm{Cl}$ NTD and the C-terminal domain (CTD) and a heterodimeric complex between the CI NTD and the MOR anti-repressor.

Furthermore, we have initiated studies of the phi13 phage genetic switch and biochemical and biophysical characterization of its $\mathrm{Cl}$, which has a similar NTD structure, but a diverse CTD. These studies help us understand the diverse molecular mechanisms of phage genetic switches, and potentially their role in transfer of antibiotic resistance. 